\title{
Research on University Library Digital Resources Integration under the Cloud Computing
}

\author{
Xinzhu Huang \\ Library of Chengdu Sport University, Chengdu, China \\ xinzhu_h@163.com
}

\begin{abstract}
With the rapid development of information technology, internet technology and communication technology, library resources building and information services of university is facing new opportunities and challenges and single library resources can not meet the needs of readers. This paper proposes a strategy to integrate a number of digital resources and attempts to explore the ways to achieve resource storage, heterogeneous data integration, resource management, etc key issues with using cloud computing technology.
\end{abstract}

Keywords- cloud computing; university library resourcel digital resources integration

\section{THE NECESSITY OF UNIVERSITY LIBRARY DIGITAL RESOURCES INTEGRATION}

This Library is the school's information center. It is not only a production base of information, but also the collecting and distribution center for a variety of information resources and an important navigation sites for information discovery, search and capture. In order to better meet the development needs of teaching, and research, regardless of the libraries of larger or other medium and small size libraries are seeking to build digital resources. With decades of development, the university library has built lots of database of e-books, enewspapers, videos, simulation test system and other types of digital information resources. However, in information surge today, with the increasing number of disciplines, interdisciplinary interpenetration and crossbar and information resources disorder and dispersed which leads to lower recall factor and pertinence factor. In addition, due to an increasingly wide range of digital resources, and in quantity, structure, distribution, dissemination, transmission means, and so has the diversity, complexity, it also leads to lower retrieval efficiency. But in the meantime, the resource requirements of readers have been continued to increase that called for a comprehensive, high-speed, convenient and accurate. Obviously, Rely on a library alone is difficult to fully meet the needs of readers. So if integrated digital library resources of libraries across the country to establish an efficient unified search platform not only can well relieve the contradiction between lack of resources and the growing literature demand for readers, but also avoid the repeatability of the library resources building effectively and improve resource utilization greatly, is very good.

\section{CONTENT OF Cloud Computing TeChNOlogy}

Cloud computing is an IT resources delivery model combined distributed computing, grid computing, parallel computing and internet to provide dynamic, scalable IT resources as a service to user via Internet ${ }^{[1]}$. The narrow computing is called IaaS (infrastructure as a service) which refers to using virtual technology to build virtual data center, and put computing and storage resources that distributed in a large number of computers and storage devices together to form a virtual pool of resources as a service to users on demand. The broader cloud computing also include SaaS (software as a service) and PaaS(platform as a service). SaaS via a browser delivering the program as a service to users and getting the service fees from them. PaaS puts the development environment, applications, database environment as a service to provide ${ }^{[2]}$. In short, cloud computing has very rich connotations, not only including service as delivery model of computing and storage infrastructure, but also including virtual hosting, application service environment renting, database environment renting, programming models, Data as a Service, Process as a Service, Application as a Service, and other modes. Cloud computing technology provides new ideas and viable technology roadmap for the integration of distributed digital resources.

Establish a digital resource platform by use of cloud computing technology putting each library's digital resources integrated centralized storage together. Then users can enjoy various digital library resources on-demand like using water, electricity in life as long as they can connect to the internet.

\section{ARCHITECTURE OF THE DigitAL RESOURCES INTEGRATION PLATFORM}

First, Digital Resources Integration use modern computer technology, network communication technology to optimize combination the digital resources according to certain needs, fuse, cluster and restructure the digital objects, functional structure and the interactions of relatively independent of each digital resource system to re-combined into a new organic whole, forming a more efficient system of digital resources system ${ }^{[3]}$.Using cloud computing technology integrated various resources to build a combined resources guarantee platform with characteristics of low-cost, strong adaptive and expandability. This system can be composed of national and provincial regional level cloud center shown in Figure 1.National level cloud center can pool Open API as in escrow provided by provincial regional level cloud center 
then serve them as PaaS or SaaS. Each library uses Main Server and App Server of local platform and integrated approach provided by cloud center to build their own cloud services platform to get resources from the cloud center. The user login to the local library with authentication method to enjoy all the resources and services provide by cloud center. The entire system use of virtual technology of cloud computing to integrated dynamic and shared hardware and software resources to maximize the input-output performance.

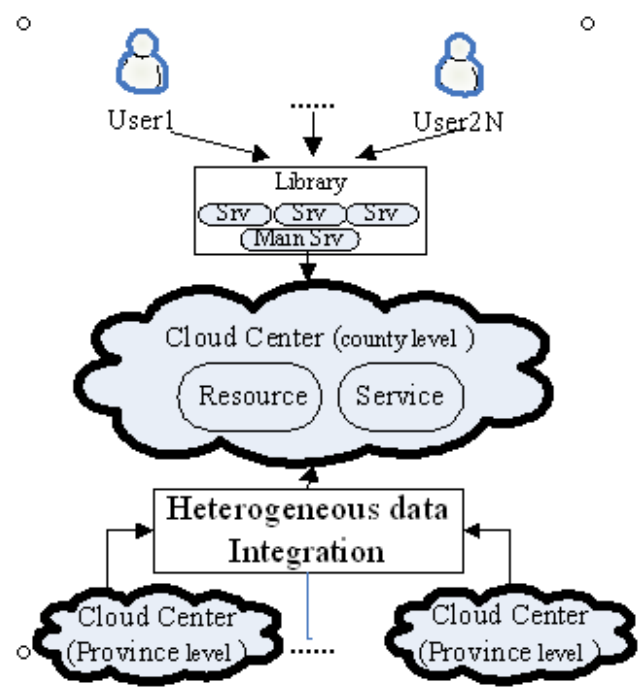

Figure 1. Architecture of the Platform

\section{Digital RESOURCE INTEGRATION PlATFORM SERVICES FRAMEWORK}

Combined with the status of digital resources in Chinese Universities, followed pragmatic, efficient and practical principles to use cloud computing technology to build cloud resources integration platform. This platform can be divided into infrastructure layer, data layer, management layer and application layer from top to bottom, such as shown in Figure $2^{[4]}$.

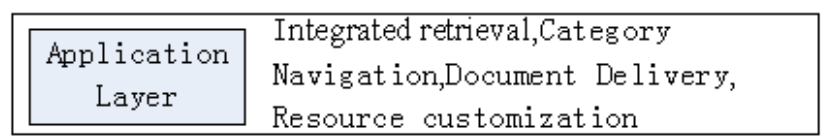

\begin{tabular}{|c|c|}
\hline $\begin{array}{c}\text { Management } \\
\text { layer }\end{array}$ & $\begin{array}{l}\text { User Management, Task Management, } \\
\text { Security Management, } \\
\text { Service monitor }\end{array}$ \\
\hline
\end{tabular}

\begin{tabular}{|l|l|}
\hline Data layer & $\begin{array}{l}\text { Dada Integrated ,Dada Analysis, Data } \\
\text { Scheduling, Data Compression, } \\
\text { Data Backup }\end{array}$ \\
\hline
\end{tabular}

\begin{tabular}{|c|c|}
\hline $\begin{array}{c}\text { Infrastructure } \\
\text { Layer }\end{array}$ & $\begin{array}{c}\text { Cluster Equipment for Storage, } \\
\text { Web-Transport Equipment etc. }\end{array}$ \\
\hline
\end{tabular}

Figure 2. Frame of the Platform

\section{A. Infrastructure Layer}

The infrastructure layer provides the basic physical storage resources, including computing, storage, data, and network devices. In order to optimize the allocation of hardware resources, use Xen, KVM, VMware and other virtual tools to abstract the underlying hardware resources to eliminate physical hardware limitations and reduce hardware management complexity through virtual and clustering technology, with the result that improved the utilization of hardware resources and ensured the scalability of cloud computing system for the unlimited storage space which growing digital resources provide, and also solve the contradiction between the data resource expansion and storage space.

\section{B. Data Layer}

Data layer using data virtual technology, shielding the distribution, heterogeneity of data source and complexity of the operation to manage and schedule heterogeneous data resources on different nodes uniformly ${ }^{[5]}$.Specific process is as follows: first of all call the Statement describing after get the user retrieval request and parsed it into logical operation of different data sources and rewrite the query requests. Then concurrency control and synergy analysis of the heterogeneous multiple data source and generate the corresponding subtasks collection. According to the task invoke a particular data source data, synthesis heterogeneous data of cloud to form a dynamic virtual environment. At last return all the data information to users what they want. Overall, the high flexibility and efficiency of Cloud computing technology fully meet the high concurrency, high load and high speed processing of massive data associated with complex multi-table retrieval searching needs for university readers.

\section{Management Layer}

At management layer, it can provide guarantee about digital resource's availability, reliability and security by clustering technology, and realize digital resource's deployment, monitoring, distribution, collaboration, fault tolerance, load balancing, fault recovery and other management functions by automatic and intelligent means. So that each university libraries can under the unified management of the cloud center,breaking the limitations of traditional campus network IP authentication mode, and making it easier to add digital resources remotely. For all the universities readers can get more valuable information than their own networked to achieve maximization of resource utilization.

\section{Application Layer}

Application layer is divided into application interface layer and application service layer. Application interface layer is to provide users with interactive interface to achieve cloud computing services ubiquitous access, including mobile phone users based on Linux, Palm, Android or Iphone system or based on Microsoft's Windows Mobile and also be a PC user through browser to access Web pages. Application service layer provides the following functions: 
1) Integrated retrieval: After a user enters a search term, through cloud computing server processing, can return related information back to user from each characteristic database, no longer need to search each database separately.

2) Category Navigation: Academic resources will be classify according to the discipline and each category revealed by knowledge element to provide users navigation of certain topic resources.The professional knowledge element linked with other digital resources interconnected to form a certain scale of knowledge networks to make digital resources more orderly, coherent and systematic.

3) Document Delivery: The information can not be fully showed online because of the intellectual property rights can be resolved by means of document delivery. Delivery may be copy and scan or by mail or e-mail based on user needs. This not only protects the important documents of intellectual property rights, but also meet the needs of readers of the special literature.

4) Resource customization: By use of RSS technology of Web2.0 technology to provide users with the $\mathrm{BSS}$ features. When readers subscribed threads resources are updated, the system will take the initiative to push relevant information to the user.

\section{CONCLUSION}

University Library digital resources integration is a difficult and long-term task that requires constant innovation and perfection. Libraries should make full use of network technology, computer technology and take advantage of strong scalable and high flexible characteristics of cloud computing technology, keep pace with the times, achieve the Omni-directional and multi-level integration of library resources to maximize library resource value.

\section{REFERENCES}

[1] Wang Changquan, Ai Fen. Study on Information Resources Integration and Service Mode Innovation of Digital Library under the Cloud Computing Environment.Library Work And Study.Vol1.pp4851.2011

[2] Libor Sarga.Cloud Computing: An Overview.Journal of Systems Integration, Vol3.pp3-14.2012.

[3] Jiang Airong Wang Ping Zheng Xiaohui.The Technical Features and Development Trends of the IntegratedManagement Systems for Distributing Heterogeneous Digital Resources--Summary about MetaLib\&SFX System.New Technology of Library and Information Service.Vol3.pp1-5.2004.

[4] LUO Jun-zhou, JIN Jia-hui, SONG Ai-bo, DONG Fang.Cloud computing: architecture and key technologies.Journal on Communications.Vol7.pp.3-18.2011.

[5] Jemila S. Hamid,Celia M.T. Greenwood,Joseph Beyene.Weighted kernel fisher discriminant analysis for integrating heterogeneous data.Computational Statistics \& Data Analysis.Vol6.pp. 20312040.2011 